\title{
Article \\ Timing Fluctuation Correction of A Femtosecond Regenerative Amplifier
}

\author{
Keyang Liu ${ }^{1,2}$, Hongyang $\mathrm{Li}^{1}$, Xinliang Wang ${ }^{1, *}$, Yanqi Liu ${ }^{1, *}$, Liwei Song ${ }^{1, * \mathbb{D}}$ and Yuxin Leng ${ }^{1}$ \\ 1 State Key Laboratory of High Field Laser Physics and CAS Center for Excellence in Ultra-Intense Laser \\ Science, Shanghai Institute of Optics and Fine Mechanics, Chinese Academy of Sciences, \\ Shanghai 201800, China; liukeyang@opt.ac.cn (K.L.); ihongyang@outlook.com (H.L.); \\ lengyuxin@siom.ac.cn (Y.L.) \\ 2 XIOPM Research Center for Attosecond Science and Technology, State Key Laboratory of Transient Optics \\ and Photonics, Xi'an Institute of Optics and Precision Mechanics, Chinese Academy of Sciences, \\ Xi'an 710119, China \\ * Correspondence: wx1@siom.ac.cn (X.W.); liuyanqi@siom.ac.cn (Y.L.); slw@siom.ac.cn (L.S.)
}

Citation: Liu, K.; Li, H.; Wang, X.; Liu, Y.; Song, L.; Leng, Y. Timing Fluctuation Correction of A Femtosecond Regenerative Amplifier Crystals 2021, 11, 1242. https:// doi.org/10.3390/cryst11101242

Academic Editor: Felice Gesuele

Received: 2 September 2021

Accepted: 28 September 2021

Published: 14 October 2021

Publisher's Note: MDPI stays neutral with regard to jurisdictional claims in published maps and institutional affiliations.

Copyright: (c) 2021 by the authors. Licensee MDPI, Basel, Switzerland. This article is an open access article distributed under the terms and conditions of the Creative Commons Attribution (CC BY) license (https:/ / creativecommons.org/licenses/by/ $4.0 /)$.

\begin{abstract}
We report on the long-term correction of a timing fluctuation between the femtosecond regenerative amplifier and the reference oscillator for the seed 100 PW laser system in the Station of Extreme Light (SEL). The timing fluctuation was characterized by a noncollinear balanced optical cross-correlator that maps the time difference to the sum frequency intensity of the amplifier and oscillator laser pulses. A feedback loop was employed to correct the timing jitter by adjusting the time delay line in the amplifier beam path. The timing fluctuation was reduced to $1.26 \mathrm{fs}$ root-mean-square from hundreds of fs over 10 hours. Benefitting from excellent performance and long-term stability, this timing jitter correction scheme, as a component of optical synchronization in the $100 \mathrm{PW}$ laser facility, will be integrated into SEL.
\end{abstract}

Keywords: timing jitter; balanced optical cross-correlator; optical synchronization; PW laser facility

\section{Introduction}

In the past few decades, the advent of the chirped pulse amplification (CPA) and optical parametric chirped pulse amplification (OPCPA) techniques has greatly promoted the development of super-intense ultrashort lasers [1,2]. So far, high-power femtosecondlevel laser systems based on CPA and OPCPA techniques have achieved an output power of several petawatts (PW), even the $10 \mathrm{PW}$ level [3-10]. In addition, there are several kilojoule-level high-energy laser facilities under construction that aim to achieve hundreds of petawatts of output and support a focus intensity of over $10^{23} \mathrm{~W} / \mathrm{cm}^{2}$, such as the Station of Extreme Light (SEL), Extreme Light Infrastructure (ELI), Exawatt Center for Extreme Light Studies (XCELS), and Optical Parametric Amplifier Line (OPAL) [11-14]. Such extreme physical conditions have opened up many significant research fields, including laboratory astrophysics, particle acceleration, nuclear physics, and fast ignition [15-18]. In all of these laser facilities, the seed laser plays a key role for subsequent amplifier lines. For example, the seed pulses are split into several parts in OPCPA and optical parametric amplification (OPA) laser systems, which are used as the signal and pre-pump pulses, respectively $[6,19,20]$. The timing between seed and pump pulses significantly influences pulse stability and conversion efficiency and should be precisely controlled. František Batysta et al. demonstrated a compact scheme that synchronized the pump and signal pulses in an OPCPA to 17 fs RMS for hours [20]. Stephan Prinz et al. reported an active pump-seed-pulse synchronization for an OPCPA system and achieved $2 \mathrm{fs}$ timing jitter between seed and pump pulses [21]. Moreover, the regenerative amplifier is indispensable to enhance pulse energy in the CPA system; the inherent principle of regenerative amplifiers implies the existence of pulse buildup time between input and output amplified pulses. 
Due to differences in regenerative amplifier architecture, the pulse buildup time ranges from hundreds of nanoseconds to a few microseconds, which corresponds to an optical path length of dozens to hundreds of meters. The timing jitter will be largely introduced by slight mechanical and pressure variations in the optical elements in the regenerative amplifier and temperature fluctuations, which negatively influence the output-time stability of the amplified pulse. In 2016, Alexis Casanova et al. reported the characterization and long-term correction of additive timing jitter between an oscillator and a regenerative amplifier [22].

In the Shanghai high repetition rate XFEL and extreme light (SHINE) facility project, the pulses of a $100 \mathrm{PW}$ laser and X-ray free-electron laser (XFEL) will be simultaneously interacted at a target for pump-probe experiments in which the synchronization of colliding pulses is a key factor. As the pulse durations of the optical laser and XFEL are both less than $20 \mathrm{fs}$, the timing jitter between them needs to be controlled precisely. Therefore, the amplifiers of the $100 \mathrm{PW}$ laser will be locked to an oscillator that is synchronized to the reference XFEL [23-25]. Moreover, CPA, OPA, and OPCPA techniques are applied in highpower laser systems. The synchronization between seed and pump pulses is important for the amplifiers. In this study, the timing jitter between the Ti:sapphire (Ti:Sa) oscillator and regenerative amplifier in a seed CPA laser system was measured by a noncollinear balanced optical cross-correlator (BOC) [22,26,27]. A home-built closed feedback loop was used to lock the timing jitter. By correcting the optical path length and optimizing the dispersion of the seed pulses, we finally decreased the timing fluctuation between the regenerative amplifier and oscillator pulses to 1.26 fs root-mean-square (RMS) over $10 \mathrm{~h}$, which implies excellent long-term stability. This timing fluctuation correction system will be integrated into the pulse synchronization system of SEL.

\section{Experimental Implementation}

The seed laser of the $100 \mathrm{PW}$ laser facility includes a CPA laser, OPA, and a gas-filled hollow-core fiber [19]. In the CPA laser system, a Ti:Sa oscillator delivered femtosecond (fs) pulses, which were split into two parts. One was sequentially stretched, amplified in a Ti:Sa regenerative amplifier, and compressed, which exported a pulse energy of $\sim 7 \mathrm{~mJ}$ with a $35 \mathrm{fs}$ pulse duration at the repetition rate of $1 \mathrm{kHz}$. The other part, with a pulse energy of $\sim 2 \mathrm{~nJ}$ at the repetition rate of $\sim 73 \mathrm{MHz}$, was used as the reference of the synchronization system in the laser facility.

The experimental setup is shown in Figure 1. The reference signal from the oscillator transmitted a multi-pass cavity (MPC) configuration to increase the beam optical path length. Subsequently, a chirped mirror pair (CMP) was employed for dispersion correction. On the other side, part of the pulses from the regenerative amplifier passed through a delay line that was controlled by a feedback circuit. As shown in the dashed box of Figure 1, the $\mathrm{BOC}$ was a noncollinear sum frequency generation (SFG) configuration, which provided background-free coincidence detection. Two pieces of type-I $\left(\theta=29.2^{\circ}, \varphi=0^{\circ}\right) \mathrm{BBO}$ crystal with a thickness of $2 \mathrm{~mm}$ were used for SFG. A 100- $\mu \mathrm{m}$-thick glass plate (GP) was inserted into one arm to introduce a time delay for the balanced detection operation. The sum frequency signals and their differential were measured by a balanced photodetector (Thorlabs PDB450). In the BOC measurement, for one arm, the sum frequency intensity was decided by the time difference between the two beams. Therefore, the relative timing fluctuation was reflected by the change in the sum frequency intensity recorded by the photodetector. Additionally, the initial intensity fluctuation of the beams was cancelled by the balance detection method. The analysis and control elements, including a PCIE data acquisition card and a feedback program, drove the delay line to correct the imbalance of the BOC. With this setting, the timing fluctuation was largely decreased by controlling the optical path of the amplifier beam. Limited by the response speed of the stepper motor (Sigma SGSP26), the feedback frequency was set to $5 \mathrm{~Hz}$, which mainly corrected the low-frequency fluctuation in the amplified pulses. 


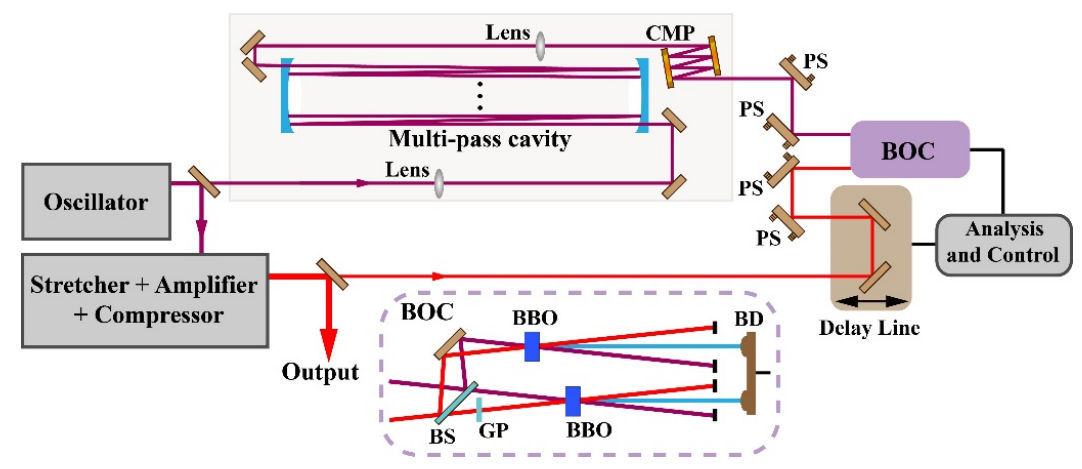

Figure 1. Experimental setup of synchronization system for the oscillator and regenerative amplifier. CMP, chirped mirror pair; PS, pointing stabilizer; BOC, balanced optical cross-correlator; BS, beam splitter; $\mathrm{GP}$, glass plate; $\mathrm{BBO}, \beta-\mathrm{BaB}_{2} \mathrm{O}_{4} ; \mathrm{BD}$, balanced photodetector.

The pulse buildup time in the regenerative amplifier was $\sim 117 \mathrm{~ns}$, which corresponded to a free space travel distance of $\sim 35 \mathrm{~m}$. To correct the optical path difference between the beams from the oscillator and the regenerative amplifier, an MPC configuration was introduced. In the MPC scheme, as shown in the light pink box in Figure 1, the focal lengths of two lens and two concave mirrors were $1 \mathrm{~m}$ and $250 \mathrm{~mm}$, respectively, and the distance between the two concave mirrors was $810 \mathrm{~mm}$. This design supported 50 roundtrip transmissions of laser pulses with a total optical path length of $\sim 40.5 \mathrm{~m}$. By applying high reflectivity (>99\%) concave mirrors, the MPC scheme achieved transmission efficiency of $65.7 \%$. Afterwards, a CMP was used to correct the dispersion of the long-distance free space transmission. Through 22 round-trip reflections, the CMP provided a dispersion correction of $-880 \mathrm{fs}^{2}$. Moreover, to eliminate interference from the environment, the lens, MPC, and CMP were all placed in an enclosed box.

\section{Results and Discussion}

At first, we did not use the MPC scheme to correct the optical path difference and sent the beams from oscillator and regenerative amplifier directly to a BOC for timing jitter measurement. The BOC signal obviously changed in a few seconds, which indicated a fast timing fluctuation. By switching on the feedback loop, the fluctuation was significantly decreased. Figure 2a illustrates the long-term measurement results of the timing fluctuation correction under closed-loop conditions, and the corresponding S-shaped optical crosscorrelation traces of the BOC (black curve) are shown in the insert. The fitting slope (red line) was $4.89 \mathrm{mv} / \mathrm{fs}$, and the timing fluctuation was locked to $2.04 \mathrm{fs}$ RMS for over $7 \mathrm{~h}$, indicating the long-term performance stability of this synchronization scheme. However, as shown in Figure $2 b$, the delay line in the feedback loop increased monotonously during the measurement, representing a unidirectional timing drift of 3.4 picosecond (ps) over $7 \mathrm{~h}$. Meanwhile, the long-term timing drift showed little correlation with environmental temperature fluctuations, which are shown in Figure 2c.

The external perturbations of the environment, such as temperature fluctuations, mechanical vibrations, and pressure variations, could influence the stability of the oscillator and the regenerative amplifier. However, in the above measurement, the timing shift was unidirectional and irrelevant to the fluctuation in environmental temperature. That means compared to the environment hazards, there was a very serious factor that caused a large and unidirectional timing drift between the oscillator and the amplifier. By measuring the arrival time of different oscillator pulses, we concluded that the timing shift was because of the change in length of the resonant cavity. A laser pulse transmitted $\sim 41 \mathrm{~m}$ in the regenerative amplifier. Meanwhile, the same pulse traveled another 10 rounds in the oscillator (with $\sim 73 \mathrm{MHz}$ repetition rate). Therefore, without optical length correction, the sum frequency made in the BOC was between the amplified pulse and the oscillator pulse, which traveled another 10 rounds in the cavity, as shown in Figure 3a. Thus, the measured 
timing shift was significantly influenced by the change in cavity length. With the change in cavity length set to $\Delta \mathrm{L}$, the resulting timing shift was $10 \times \Delta \mathrm{L} / \mathrm{c}$. A timing shift of $3.4 \mathrm{ps}$, shown in Figure $2 b$, could be caused by a $\Delta \mathrm{L}$ of $102 \mu \mathrm{m}$. During a period of $7 \mathrm{~h}$, changes of this magnitude were possible in the cavity.
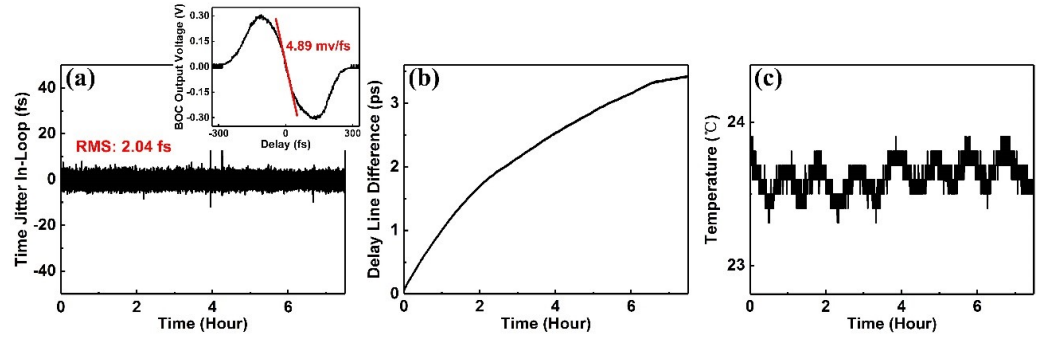

Figure 2. Timing fluctuation correction without optical path length correction: (a) timing jitter between the oscillator and the regenerative amplifier with the feedback loop closed (the insert shows optical cross-correlation traces); (b) variation of the delay line in the feedback loop; (c) temperature fluctuations in the environment.

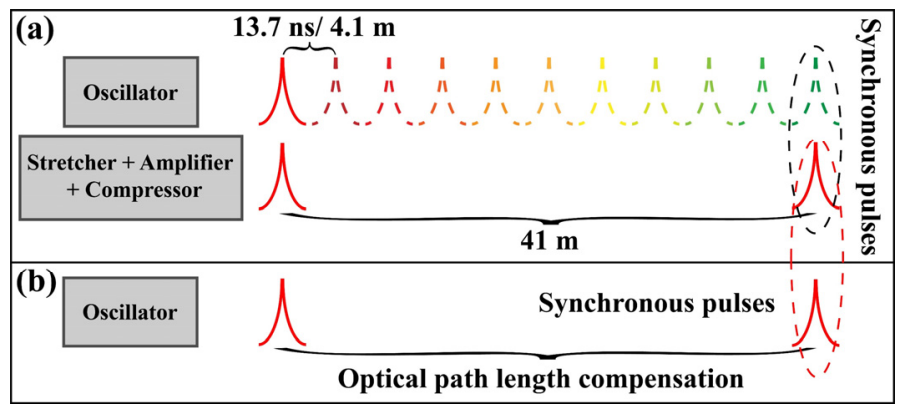

Figure 3. Synchronous pulses (a) without and (b) with optical path correction (curves in different colors represent different laser pulses).

After correction of the optical path length, a sum frequency was achieved between the regenerative amplified pulse and the oscillator pulse without further round trips in the cavity, as shown in Figure 3b. The synchronization was no longer influenced by the change in cavity length. The long-term timing jitter under the closed-loop condition, change in delay line, and temperature variation is shown in Figure 4. The timing jitter between oscillator and regenerative amplifier pulses was locked to $1.26 \mathrm{fs}$ RMS for over 10 hours, as shown in Figure 4a. The insert in Figure 4a indicates S-shaped optical crosscorrelation traces of BOC (black curve) and corresponding fitting slope (red line) with $7.5 \mathrm{mv} / \mathrm{fs}$. Owing to the perfect dispersion correction by the CMP (for oscillator pulses) and the grating compressor (for regenerative amplifier pulses), the laser pulse durations of the two beams were both shorter than $100 \mathrm{fs}$, which ensured high intensity of the sum frequency and excellent sensitivity of the BOC. The long-term stable performance of this synchronization scheme was confirmed. The change in delay line (Figure $4 \mathrm{~b}$ ) and environmental temperature (Figure 4c) followed a similar trend, which implied that the timing jitter was actually affected by environmental temperature. The change in the delay line also indicated the timing fluctuation without the feedback loop, which showed a timing shift from $-200 \mathrm{fs}$ to $100 \mathrm{fs}$, as illustrated in Figure $4 \mathrm{~b}$. Therefore, the feedback loop decreased the timing fluctuation from hundreds of fs to $1.26 \mathrm{fs}$ over 10 hours; in other words, the timing stability was improved by two orders of magnitude. In the scheme, the feedback frequency was limited by the delay stage, and timing jitter higher than $5 \mathrm{~Hz}$ could not be corrected by the current setup. Moreover, the timing accuracy was also limited by the BOC measurement, the accuracy of which was affected by the pulse duration. This was an effective design to correct the low frequency timing jitter of the amplifier within the 
laboratory environment, e.g., temperature fluctuation within $\pm 0.5{ }^{\circ} \mathrm{C}$ and air flow-induced vibration lower than $5 \mathrm{~Hz}$.
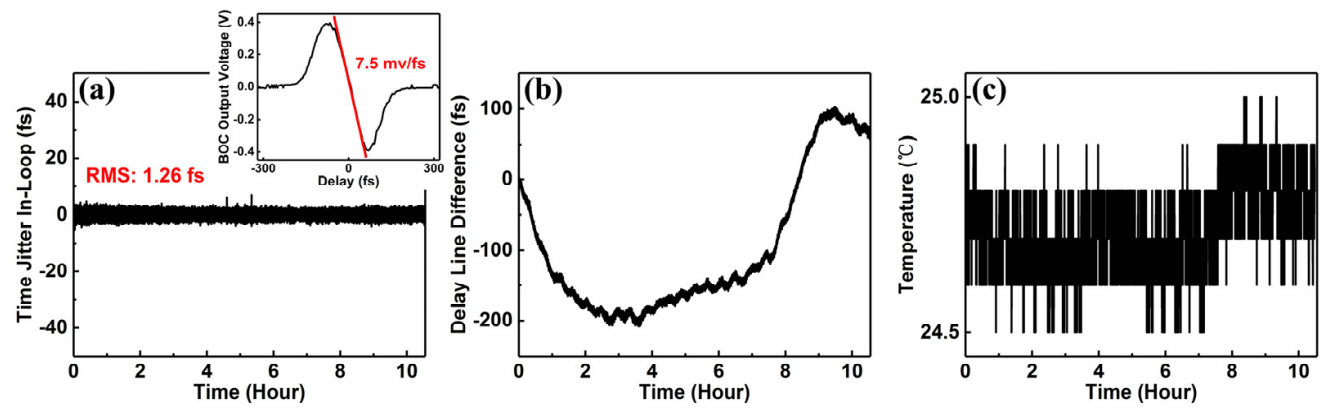

Figure 4. Timing fluctuation correction with optical path length correction: (a) timing jitter between oscillator and regenerative amplifier pulses (insert shows the " $\mathrm{S}$ " curve of the BOC measurement); (b) change in the delay line, (c) environment temperature fluctuation.

\section{Conclusions}

In summary, we demonstrated a synchronization scheme for the seed laser of the 100 PW laser facility in SEL. The timing fluctuation between the oscillator and regenerative amplifier pulses was reduced to 1.26 fs RMS over 10 hours. The timing jitter was measured by a noncollinear BOC and corrected by a feedback loop. To avoid influence from the oscillator cavity change, an MPC was employed to correct the optical path length difference between the oscillator and amplifier. A CMP configuration was used to correct the laser pulse dispersion introduced by long distance transmission and multiple reflections. By utilizing this system, precise long-term synchronization was achieved in the front end of the 100 PW laser facility. In the future, the system will be upgraded for the synchronization of the 100 PW laser and a reference oscillator, thus offering the possibility for high-power laser and XFEL pump-probe experiments.

Author Contributions: Conceptualization, K.L. and L.S.; methodology, K.L.; software, Y.L. (Yuxin Leng); validation, K.L., L.S. and X.W.; formal analysis, L.S.; investigation, H.L.; resources, Y.L. (Yanqi Liu); data curation, K.L.; writing-original draft preparation, K.L. and L.S.; writing-review and editing, L.S. and X.W.; funding acquisition, L.S. and Y.L. (Yanqi Liu) All authors have read and agreed to the published version of the manuscript.

Funding: This research was funded by the Shanghai high repetition rate XFEL and extreme light facility (SHINE) project; 100 Talents Program of CAS; Shanghai Pujiang Program (19PJ1410500).

Data Availability Statement: The data presented in this study are available on request from the corresponding author. The data are not publicly available due to the project regulations.

Conflicts of Interest: The authors declare no conflict of interest.

\section{References}

1. Strickland, D.; Mourou, G. Compression of amplified chirped optical pulses. Opt. Commun. 1985, 56, 219-221. [CrossRef]

2. Dubietis, A.; Jonušauskas, G.; Piskarskas, A. Powerful femtosecond pulse generation by chirped and stretched pulse parametric amplification in BBO crystal. Opt. Commun. 1992, 88, 437-440. [CrossRef]

3. Aoyama, M.; Yamakawa, K.; Akahane, Y.; Ma, J.; Inoue, N.; Ueda, H.; Kiriyama, H. 085-PW, 33-fs Ti:sapphire laser. Opt. Lett. 2003, 28, 1594-1596. [CrossRef] [PubMed]

4. Xu, L.; Yu, L.; Liang, X.; Chu, Y.; Hu, Z.; Ma, L.; Xu, Y.; Wang, C.; Lu, X.; Lu, H.; et al. High-energy noncollinear optical parametric-chirped pulse amplification in LBO at $800 \mathrm{~nm}$. Opt. Lett. 2013, 38, 4837-4840. [CrossRef] [PubMed]

5. Sung, J.H.; Lee, H.W.; Yoo, J.Y.; Yoon, J.W.; Lee, C.W.; Yang, J.M.; Son, Y.J.; Jang, Y.H.; Lee, S.K.; Nam, C.H. 42 PW, 20 fs Ti:sapphire laser at $01 \mathrm{~Hz}$. Opt. Lett. 2017, 42, 2058-2061. [CrossRef]

6. Zeng, X.; Zhou, K.; Zuo, Y.; Zhu, Q.; Su, J.; Wang, X.; Wang, X.; Huang, X.; Jiang, X.; Jiang, D.; et al. Multi-petawatt laser facility fully based on optical parametric chirped-pulse amplification. Opt. Lett. 2017, 42, 2014-2017. [CrossRef] 
7. le Garrec, B.; Papadopoulos, D.N.; le Blanc, C.; Zou, J.P.; Chériaux, G.; Georges, P.; Druon, F.; Martin, L.; Fréneaux, L.; Beluze, A.; et al. Design update and recent results of the Apollon 10 PW facility. Proc. SPIE 2017, 10238, 102380Q.

8. Li, W.; Gan, Z.; Yu, L.; Wang, C.; Liu, Y.; Guo, Z.; Xu, L.; Xu, M.; Hang, Y.; Xu, Y.; et al. 339 J high-energy Ti:sapphire chirped-pulse amplifier for $10 \mathrm{PW}$ laser facility. Opt. Lett. 2018, 43, 5681-5684. [CrossRef]

9. Lureau, F.; Matras, G.; Chalus, O.; Derycke, C.; Morbieu, T.; Radier, C.; Casagrande, O.; Laux, S.; Ricaud, S.; Rey, G.; et al. High-energy hybrid femtosecond laser system demonstrating $2 \times 10 \mathrm{PW}$ capability. High Power Laser Sci. Eng. $2020,8,43$. [CrossRef]

10. Danson, C.N.; Haefner, C.; Bromage, J.; Butcher, T.; Chanteloup, J.C.-F.; Chowdhury, E.A.; Galvanauskas, A.; Gizzi, L.A.; Hein, J.; Hillier, D.I.; et al. Petawatt and exawatt class lasers worldwide. High Power Laser Sci. Eng. 2019, 7, 7. [CrossRef]

11. Cartlidge, E. The light fantastic. Science 2018, 359, 382-385. [CrossRef]

12. Zuegel, J.D.; Bahk, S.W.; Begishev, I.A.; Bromage, J.; Dorrer, C.; Okishev, A.V.; Oliver, J.B. Status of high-energy OPCPA at LLE and future prospects. In Proceedings of the 2014 Conference on Lasers and Electro-Optics (CLEO)_Laser Science to Photonic Applications, San Jose, CA, USA, 8-13 June 2014; pp. 1-2. [CrossRef]

13. Shaykin, A.; Kostyukov, I.; Sergeev, A.; Khazanov, E. Prospects of PEARL 10 and XCELS Laser Facilities. Rev. Laser Eng. 2014, 42, 141. [CrossRef]

14. Cartlidge, E. Eastern Europe's laser centers will debut without a star. Science 2017, 355, 785. [CrossRef] [PubMed]

15. Kodama, R.; Norreys, P.A.; Mima, K.; Dangor, A.E.; Evans, R.G.; Fujita, H.; Kitagawa, Y.; Krushelnick, K.; Miyakoshi, T.; Miyanaga, N.; et al. Fast heating of ultrahigh-density plasma as a step towards laser fusion ignition. Nat. Cell Biol. 2001, 412, 798-802. [CrossRef] [PubMed]

16. Mourou, G.A.; Tajima, T.; Bulanov, S.V. Optics in the relativistic regime. Rev. Mod. Phys. 2006, 78, 309-371. [CrossRef]

17. Fujioka, S.; Takabe, H.; Yamamoto, N.; Salzmann, D.; Wang, F.; Nishimura, H.; Li, Y.; Dong, Q.; Wang, S.; Zhang, Y.; et al. X-ray astronomy in the labora-tory with a miniature compact object produced by laser-driven implosion. Nat. Phys. 2009, 5, 821-825. [CrossRef]

18. Daido, H.; Nishiuchi, M.; Pirozhkov, A. Review of laser-driven ion sources and their applications. Rep. Prog. Phys. 2012, 75, 056401. [CrossRef]

19. Shao, B.; Li, Y.; Peng, Y.; Wang, P.; Qian, J.; Leng, Y.; Li, R. Broad-bandwidth high-temporal-contrast carri-er-envelope-phasestabilized laser seed for 100 PW lasers. Opt. Lett. 2020, 45, 2215-2218. [CrossRef]

20. Batysta, F.; Antipenkov, R.; Green, J.T.; Naylon, J.A.; Novák, J.; Mazanec, T.; Hříbek, P.; Zervos, C.; Bakule, P.; Rus, B. Pulse synchronization system for picosecond pulse-pumped OPCPA with femtosecond-level relative timing jitter. Opt. Express 2014, 22, 30281-30286. [CrossRef]

21. Prinz, S.; Häfner, M.; Schultze, M.; Teisset, C.Y.; Bessing, R.; Michel, K.; Kienberger, R.; Metzger, T. Active pump-seed-pulse synchronization for OPCPA with sub-2-fs residual timing jitter. Opt. Express 2014, 22, 31050-31056. [CrossRef]

22. Casanova, A.; D'Acremont, Q.; Santarelli, G.; Dilhaire, S.; Courjaud, A. Ultrafast amplifier additive timing jitter character-ization and control. Opt. Lett. 2016, 41, 898-900. [CrossRef] [PubMed]

23. Xin, M.; Şafak, K.; Kärtner, F.X. Ultra-precise timing and synchronization for large-scale scientific instruments. Optica 2018, 5, 1564-1578. [CrossRef]

24. Xin, M.; Şafak, K.; Peng, M.Y.; Callahan, P.T.; Kärtner, F.X. One-femtosecond, long-term stable remote laser synchronization over a 35-km fiber link. Opt. Express 2014, 22, 14904-14912. [CrossRef] [PubMed]

25. Şafak, K.; Xin, M.; Callahan, P.T.; Peng, M.Y.; Kartner, F.X. All fiber-coupled, long-term stable timing distribution for free-electron lasers with few-femtosecond jitter. Struct. Dyn. 2015, 2, 041715. [CrossRef]

26. Kim, J.; Chen, J.; Cox, J.; Kärtner, F.X. Attosecond-resolution timing jitter characterization of free-running mode-locked lasers. Opt. Lett. 2007, 32, 3519-3521. [CrossRef]

27. Kärtner, F.; Wong, F.; Kim, J. Compact Background-Free Balanced Cross-Correlators. U.S. Patent 7,940,390 B2, 10 May 2011. 